\title{
QUELQUES REMARQUES SUR LE VOCABULAIRE QUI S'AJUSTE AU MONDE CONTEMPORAIN
}

\author{
Elżbieta PACHOCIŃSKA \\ Université de Varsovie
}

\begin{abstract}
This article presents the vocabulary significant to our contemporary world in 2016 and 2017. It has been used in connection with the most important political, social, economic and cultural transformations.
\end{abstract}

\section{INTRODUCTION}

Nous voulons réfléchir sur le vocabulaire qui nous permet de saisir la signification du monde dans lequel nous vivons actuellement. Il s'agit des mots motivés par l'actualité politique, sociale, culturelle et économique des dernières années, en particulier des années 2016 et 2017. Nous allons commencer par le vocabulaire qui nous semble significatif pour décrire le renouveau du mensonge dans l'espace public, ensuite nous passerons aux mots qui témoignent de l'impact des changements sociaux, économiques et civilisationnels.

\section{LE VOCABULAIRE DE LA DÉSINFORMATION}

Le vocabulaire désignant la désinformation ne renvoie pas seulement aux informations volontairement erronées ou trompeuses qui circulent dans les médias et sur les réseaux sociaux, mais il signale aussi un phénomène social présent un peu partout dans le monde. Il s'agit de la crise moderne de confiance dans les faits présentés par les élites politiques, les partis et les médias traditionnels qui ont perdu de l'autorité pour un électorat important. Cette réalité nouvelle est une 
conséquence de l'avènement en politique des gens qui ne respectent plus les règles du politiquement correct et de la responsabilité en politique. Certains leaders préfèrent flatter la sensibilité, les préjugés et les ressentiments de leurs électeurs que leur dire la vérité. Pour lutter contre le fléau des fausses informations qui inondent les réseaux sociaux, les journalistes ont créé le vocabulaire au service de la bonne cause en appelant ces informations intox, ils ont aussi dépoussiéré le vieux vocabulaire, tel hoax, bobard (fam.) pour ne pas fâcher l'Académie française qui conseille d'employer les synonymes, tels bobard, boniment, contrevérité, mensonge, ragot, tromperie, trucage ${ }^{1}$ au lieu de l'anglicisme fake-news. Mais en même temps les personnes publiques ont trouvé les euphémismes, tels faits alternatifs pour cacher leurs mensonges. Ainsi, comme le disent certains commentateurs politiques, nous sommes entrés dans l'ère de la post-vérité. L'usage a montré que c'est la fake news et l'intox qui sont le plus fréquemment employés pour désigner les facettes du mensonge. Nous allons commencer par l'expression fake news parce qu'elle a profondément marqué le début de l'année 2017 à la suite de l'investiture du président américain Donald Trump.

\subsection{LA FAKE NEWS}

Le dictionnaire britannique Collins vient d'élire fake news comme mot de l'année 2017, bien que cette locution soit apparue dans les années 1990 à la télévision américaine pour décrire « de fausses nouvelles, en général sensationnelles, déguisées en vraies informations $»^{2}$ selon le Collins. Elle devient incontournable pendant deux événements politiques qui ont eu lieu en 2016 : la dernière campagne électorale aux États-Unis et la campagne sur le Brexit au Royaume-Uni ${ }^{3}$. Mais c'est Donald Trump, le président des États-Unis, qui a particulièrement contribué à sa popularité. Il l'employait à rebours pour dénoncer comme les fake news, les informations sur lui qu'il jugeait mensongères, colportées par des " médias malhonnêtes ». En première ligne, il a pointé le New York Times et le Washington Post, les journaux qui parmi les professionnels du journalisme jouissent d'une grande estime ${ }^{4}$.

1 http://www.lefigaro.fr/langue-francaise/actu-des-mots/2017/11/02/37002-20171102ARTFIG00264 -fake-news-elu-mot-de-l-annee-par-le-dictionnaire-collins.php (dernière consultation : le 26.11.2017).

${ }^{2}$ Ibid.

${ }^{3}$ Brexit est une abréviation de British Exit, évoquant la sortie du Royaume-Uni de l'Union européenne. Le 23 juin 2016, lors d'un référendum organisé par l'ancien Premier ministre David Cameron, 51,9\% des Britanniques ont choisi de quitter l'UE.

4 http://www.lefigaro.fr/actualite-france/2017/03/06/01016-20170306ARTFIG00187-fake-newsun- meme-terme-pour-plusieurs-realites.php (dernière consultation : le 26.11.2017). 
Au moment où le mot commence à circuler dans l'espace public, il est employé pour dénoncer des manipulations variées. Tout d'abord, le néologisme fake news $\mathrm{s}^{5}$ ne désigne pas un article faux, au sens d'inexact, mais une publication qui fait semblant d'être un article de presse professionnelle. Son but est de tromper les internautes et de leur faire passer une désinformation comme une information vraie. D'un côté, il s'agit des sites internet qui imitent des médias professionnels, par conséquent ils ne respectent aucune déontologie journalistique en postant des articles, des informations, des vidéos, etc. volontairement trompeurs. Paradoxalement, ce sont des sites particulièrement partagés sur les réseaux sociaux à cause de la thématique qu'ils traitent, à savoir des sujets sensationnels et conspirationnistes. D'un autre côté, ces sites visent à « attirer la confiance et le ralliement des internautes avides de contre-discours » par des articles politiquement engagés et aussi à « décrédibiliser les médias traditionnels et adversaires politiques, en instituant méfiance et relativisme à leur égard $»^{6}$. Il arrive aussi que les informations fausses soient introduites par des médias satiriques qui mettent en circulation leurs railleries et critiques en les présentant comme sérieuses ${ }^{7}$. Bref, on qualifie de fake news de vrais articles erronés et de faux articles imitant de vrais articles.

Dans le contexte des dernières élections présidentielles aux États-Unis et en France, les médias présentaient certaines fake news comme une arme dans la guerre hybride de la Russie qui voulait ainsi influencer les événements politiques dans le monde ${ }^{8}$.

\subsection{L'INTOXE}

Le $T L F i^{9}$ et le dictionnaire du CNRTL en ligne ${ }^{10}$ donnent les mêmes informations :

Intox, Intoxe, subst. fém., abrév. fam. Action psychologique. Synon. matraquage. Campagne systématique de mise en condition de l'opinion publique par la diffusion d'opinions tantôt vraies tantôt fausses et plus ou moins alarmantes.

${ }^{5}$ La langue anglaise distingue en effet ce qui est false (faux au sens d'erroné) de ce qui est fake (faux au sens d'une imitation).

${ }^{6}$ On cite parmi les sites les plus « emblématiques des fake news de l'année 2016 » Empire News, NBC.com.co aux États-Unis et Minute ou Rivarol en France. Source : http://www.lefigaro. fr/actualite-france/2017/03/06/01016-20170306ARTFIG00187-fake-news-un-meme-terme-pourplusieurs-rea lites.php (dernière consultation : le 26.11.2017).

${ }^{7}$ P. ex. Le Gorafi en France, Nordpresse en Belgique, The Onion aux États-Unis.

$8 \mathrm{https} / /$ www.rts.ch/info/monde/8551111-1'influences-des-fake-news-sur-la-presidentielle-francais e-serait-reelle-html (dernière consultation : le 24.11.2017).

${ }^{9} \mathrm{http}: / /$ atilf.atilf.fr.

${ }^{10} \mathrm{http}: / / \mathrm{www} . \mathrm{cnrtl} . \mathrm{fr}$. 
Le mot a pris le sens de mensonge après les émissions de télévision « Double jeu » diffusées en 1991 et 1992 sur France 2, où les invités devaient se prononcer si les reportages présentés étaient vrais ou faux ${ }^{11}$. Aujourd'hui, ce mot est déjà bien installé dans le vocabulaire des journalistes français qui, en s'inspirant de leurs collègues américains, ont créé le genre journalistique nouveau appelé le factchecking. Depuis fin 2008, les articles de fact-checkeurs sont publiés par le journal Libération dans la rubrique Désintox. En 2014, c'est la rubrique Les Décodeurs qui fait son apparition dans le journal Le Monde. La technique journalistique de ces deux journaux est la même. On confronte l'information erronée (« intox ») qui circule dans les médias avec les faits. Ainsi, on décrypte son caractère fallacieux. Il est à observer que le mot apparaissait souvent pendant les dernières élections présidentielles en France, surtout en référence aux discours de Marine Le Pen, candidate du Front National.

\subsection{LES FAITS ALTERNATIFS}

Cette expression a été utilisée pour la première fois dans un contexte bien précis. C'est la conseillère de D. Trump à la Maison Blanche qui en est l'auteure ${ }^{12}$. Elle l'a employée en voulant justifier la contre-vérité du porte-parole du président des États-Unis que "sa cérémonie d'investiture fut la plus grande en termes d'audience ». Ainsi, le porte-parole voulait nier le fait que pendant la même cérémonie du président précédent Barack Obama en 2009 la foule était beaucoup plus nombreuse. Cela n'a pas découragé le porte-parole d'affirmer lors d'une conférence de presse : « parfois nous pouvons être en désaccord avec les faits $»^{13}$.

L'expression fait alternatif a été très critiquée par la presse américaine, qui $\mathrm{y}$ voit à la fois une stratégie pour discréditer les médias comme contre-pouvoir et une menace pour la démocratie. «Tout ce que nous pouvons faire est de monter à la barre et le dire de manière simple et sans équivoque : un mensonge, c'est un mensonge, c'est un mensonge ! », a souligné sur Facebook le journaliste Dan Rather $^{14}$.

\footnotetext{
${ }^{11} \mathrm{https}$ //fr.wiktionary.org/wiki/intox (dernière consultation : le 24.11.2017).

${ }^{12}$ Il s'agit de Kellyanne Conway qui justifiait les paroles de Sean Spicer, le porte-parole du Président D. Trump : http://www.lemonde.fr/les-decodeurs/article/2017/01/25/faits-alternatifsfake-news-post-verite petit-lexique-de-la-crise-de-l-information_5068848_4355770.html (dernière consultation : le 20.11.2017).

${ }^{13}$ Ibid.

${ }^{14} \mathrm{Ibid}$.
} 


\subsection{LA POST-VÉRITÉ}

Post-truth (post-vérité) a été choisi comme mot de l'année 2016 par Oxford English Dictionary (2017). Pourtant l'expression est apparue pour la première fois dans le livre de Ralph Keyes, The Post-Truth Era: Dishonesty and Deception in Contemporary Life en 2004 aux États-Unis. Un an après, le comédien américain Stephen Colbert a popularisé le mot truthiness dans le sens que post-vérité a acquis aujourd'hui, c'est-à-dire "une vérité subjective, propre à chaque individu ». Mais ce n'est qu'en 2016 que l'expression a connu son succès dans les médias mondiaux. Selon les dictionnaires Oxford, c'est une « caractéristique générale de notre époque $»^{15}$.

Cet adjectif fait référence « à des circonstances dans lesquelles les faits objectifs ont moins d'influence pour modeler l'opinion publique que les appels à l'émotion et aux opinions personnelles $\gg{ }^{16}$. En suivant toujours les commentaires du dictionnaire, on apprend que post-vérité est devenu en 2016 « un pilier du commentaire politique », surtout dans le contexte du référendum sur le Brexit au Royaume-Uni où les mensonges et exagérations ont circulé abondamment, en particulier dans le camp pro-Brexit. Le même phénomène est à observer pendant l'élection présidentielle aux États-Unis ${ }^{17}$. Le succès de ce néologisme reflète un phénomène social nouveau, c'est-à-dire la méfiance croissante des gens vis-à-vis des faits présentés par les politiques et les médias dits traditionnels. Dans le communiqué des éditeurs du dictionnaire, on peut lire :

Plutôt que de faire simplement référence à une période postérieure à une situation spécifique ou à un événement comme dans " après-guerre ", le préfixe post- dans post-vérité a une signification proche de l'idée « d'appartenir à une période dans laquelle le concept spécifié est devenu sans importance $»^{18}$.

Wikipedia note aussi qu'en France, l'expression ère post-vérité (post-truth politics) est fréquemment utilisée par les commentateurs politiques dès 2016,

${ }^{15} \mathrm{https} / / /$ www.lesechos.fr/16/11/2016/lesechos.fr/0211500050190_selon-les-dictionnaires-oxfor d--le-mot-de-l-annee-est---.htm (dernière consultation : le 20.11.2017).

${ }^{16} \mathrm{http}: / /$ next.liberation.fr/culture-next/2016/11/16/post-verite-mot-de-1-annee-du-dictionnaire-ox ford_1528775 (dernière consultation : le 20.11.2017).

${ }^{17}$ P. ex. « l'argument de la campagne pro-Brexit qui affirmait que le Royaume-Uni versait à l'Europe 350 millions de livres sterling par semaine, et que cet argent pourrait être rendu aux services de santé en cas de retrait de l'Union européenne », http://tempsreel.nouvelobs.com/monde/20160914. OBS8003/brexit-trump-bienvenue-dans-l-ere-post-verite.html (dernière consultation : le 21.11.2017).

${ }^{18} \mathrm{http}: / /$ next.liberation.fr/culture-next/2016/11/16/post-verite-mot-de-l-annee-du-dictionnaire-ox ford_1528775 (dernière consultation : le 20.11.2017). 
au point d'entrer un an après dans Le Petit Larousse et Le Robert illustré. On remarque que l'expression synonyme ère post-factuelle (post-factual politics) est aussi mise en circulation dans les médias ${ }^{19}$. Le néologisme post-vérité figure parmi les mots de l'année 2017 en France choisi par les internautes ${ }^{20}$.

\subsection{LE HOAX}

Ce n'est pas un vrai néologisme, mais cet anglicisme a acquis aujourd'hui un sens particulier. Il désigne un canular diffusé le plus souvent par le courriel, canular $d u$ web, des rumeurs répandues de manière virale par des chaînes de mail, diffusant fausses informations alarmistes, appels à dons malintentionnés ou désinformation assumée ${ }^{21}$.

Selon l'Oxford Dictionary, le hoax remonterait au moins au XVIII' siècle, il dériverait de hocus pocus qui est l'abréviation de hac pax max deus admix, parodie de la formule magique en pseudo-latin née au XVII ${ }^{\mathrm{e}}$ siècle, et qui servait, déjà, à jouer des tours et à tromper l'auditoire.

\section{Le MOT PRÉfÉRÉ D'EMmANUEl MaCron}

Emmanuel Macron, le nouveau président de France, s'est déjà fait remarquer par son vocabulaire optimiste. Les internautes ont choisi comme mot de l'année 2017 le renouveau qui a marqué l'élection présidentielle en 2017, mais le jury a opté pour renouvellement. D'autres mots sur lesquels le jury débattait étaient : Brexit, hésitation, clivant, promesses, post-vérité, posture, fictif, colère, renouveau, populisme, auxquels s'ajoutait le mot matraque, élu par collégiens et lycéens de la Nièvre. Comme l'expliquait le président du jury Roland Cayrol :

[le jury] a préféré [...] choisir l'optimisme, plutôt que les dangers menaçant la démocratie. Mais plutôt que " renouveau », au parfum politique un peu suranné - et qui aujourd'hui reste à prouver, il a opté

${ }^{19} \mathrm{https} / / /$ fr.wikipedia.org/wiki/\%C3\%88re_post-v\%C3\%A9rit\%C3\%A9 (dernière consultation : le 26.11.2017).

${ }^{20} \mathrm{https}$ //fr.wikipedia.org/wiki/Hoax, http://www.lemonde.fr/les-decodeurs/article/2017/01/25/ faits-alternatifs-fake-news-post-verite-petit-lexique-de-la-crise-de-1-information_5068848_4355770. htm (dernière consultation : le 26.11.2017).

${ }^{21}$ Ibid. 
pour RENOUVELLEMENT. C'est bien un renouvellement (des idées, des pratiques, des visages) qui est aujourd'hui à l'ordre du jour. En espérant qu'il conduira à un vrai renouveau de la démocratie ${ }^{22}$.

Nous avons trouvé dans un des discours d'Emmanuel Macron ${ }^{23}$ neuf occurrences de renouvellement employé dans deux sens principaux. Le premier sens articule le fait que c'est lui, son gouvernement et les députés d'En Marche qui sont porteurs de renouvellement par le fait même de ne jamais exercer de fonctions publiques. Voici un fragment qui l'illustre :

Le sel même de notre République est de savoir conjuguer ces exigences. De faire tout cela, en quelque sorte, " en même temps ». Cette voie désoriente tous ceux qui s'étaient habitués à faire carrière sur les schémas anciens. Il en est ainsi à chaque période de renouveau et nous n'avons pas à nous en inquiéter. Mais nous avons à prendre la mesure des efforts que va nous imposer cette formidable soif de renouvellement dont nous sommes, vous et moi, les porteurs ${ }^{24}$.

Le deuxième sens est celui de 'changement' :

[...] nous limiterons le cumul des mandats dans le temps pour les parlementaires. Car il s'agit là de la clef de voûte d'un renouvellement qui ne se produira pas sous la pression de l'exaspération citoyenne mais deviendra le rythme normal de la respiration démocratique ${ }^{25}$.

\section{L'IMPACT DES CHANGEMENTS SOCIO-ÉCONOMIQUES}

Le changement de notre société est marqué par la nouvelle expression écriture inclusive qui fait son entrée dans Le Petit Larousse 2017. Elle est accompagnée de féminisme ${ }^{26}$, mot de l'année 2017 aux États-Unis, motivé par l'actualité, à savoir les protestations des femmes après les attaques verbales de Donald Trump et les agressions sexuelles du grand producteur à Hollywood, Harvey Weinstein.

\footnotetext{
${ }^{22} \mathrm{http} / / /$ motdelannee.fr (dernière consultation : le 30.11.2017).

${ }^{23}$ Il s'agit du discours prononcé devant deux Chambres du Parlement réunies en Congrès le 3 juillet 2017 à Versailles. Source : http://www.bfmtv.com/politique/texte-le-discours-d-emmanuelmacron-au-congres-de-versailles-1200523.html (dernière consultation : le 30.11.2017).

${ }^{24}$ Ibid.

${ }^{25}$ Ibid.

${ }^{26}$ Élu par le dictionnaire Merriam-Webster, équivalent amércain du Petit Robert.
} 


\subsection{LA LANGUE FONDÉE SUR L'ÉGALITÉ : ÉCRITURE INCLUSIVE}

L'adjectif inclusif est employé dans le sens logique et/ou grammatical. Par exemple, le dictionnaire CNRTL en ligne en donne les acceptions suivantes :

[Dans une série ou un ensemble défini] Qui enferme, qui comprend quelque chose en soi. Anton. exclusif.

LING. (Personne) inclusive : Première personne du pluriel affectée dans certaines langues d'une forme propre, telle que dans le collectif du type " nous » on comprend, avec la ou les personnes qui parlent, celle ou celles à qui on s'adresse ${ }^{27}$.

Dans le Manuel d'écriture inclusive, le sous-titre invite les lecteurs à faire progresser l'égalité femmes/hommes par sa manière d'écrire (Haddad, 2017) ${ }^{28}$. Le manuel se réfère aux multiples travaux de linguistes, grammairiens et chercheurs qui portent sur « la qualité éthique de la langue, c'est-à-dire à sa faculté d'être discriminante, dévalorisante ou égalitaire ou non sexiste ou raciste », écrit dans la postface A.-M. Houdebine (ibid., p. 17).

Le manuel préconise trois règles d'écriture inclusive :

- l'accord en genre des noms de fonctions, grades, métiers et titres ;

- l'usage du féminin et du masculin, que ce soit par l'énumération ou par ordre alphabétique (elle et il), l'usage d'un point milieu (acteur-ricess), ou le recours aux termes épicènes ;

- l'usage des antonomases du nom commun femme et homme est déconseillé, par exemple, il est mieux de dire «droits Humains » (ou « droits humains ») que « droits de l'Homme».

\section{LE MOT CLÉ DU CHANGEMENT ÉCONOMIQUE : UBÉRISATION}

Le mot témoin du changement économique est ubérisation. Pour Bernard Cerquiglini (2017) ${ }^{29}$ c'est le mot de l'année 2017. Ce néologisme a été popularisé par Maurice Lévy, patron de Publicis, qui l'a employé lors d'une interview accordée en décembre 2014 au Financial Times. Le terme est créé à partir du nom de l'entreprise californienne Uber connue pour ses méthodes innovantes qui ont bouleversé la structure économique traditionnelle. Il s'agit de mettre en relation

\footnotetext{
${ }^{27} \mathrm{http}: / /$ www.cnrtl.fr.

${ }^{28}$ www.ecriture-inclusive.fr (dernière consultation : le 28.12.2017).

${ }^{29} \mathrm{https}: / / w w w . f r a n c e i n t e r . f r / e m i s s i o n s / 1-i n v i t e-d e-8 h 20 / 1-i n v i t e-d e-8 h 20-22-d e c e m b r e-2017$ (dernière consultation : le 29.12.2017).
} 
les entreprises et leurs clients à travers une plateforme numérique en évitant ainsi les intermédiaires. Par conséquent, les services deviennent moins chers. Uber s'est fait connaître en France avec la révolte des taxis dénonçant une concurrence déloyale, par des chauffeurs amateurs sans licence professionnelle qui proposaient des tarifs particulièrement bas en prenant contact directement avec les clients à travers une application sur leurs téléphones portables. Le terme ubérisation a depuis connu un vif essor dans la sphère médiatique et connote pour les uns des pratiques déloyales, mais pour les autres une innovation, une transformation dans l'intérêt de l'usager. On parle alors d'un phénomène global d'ubérisation de la société et d'économie du partage.

Son nom symbolise désormais le lien entre les nouvelles technologies et la précarisation, mais aussi les dérégulations sociales au vu du droit du travail qui n'est pas appliqué surtout au niveau de la protection sociale ou des congés payés. Les craintes des entreprises et des salariés se voient dans les titres des journaux français :

" L'ubérisation peut-elle mettre en danger l'économie ? », « L'ubérisation galopante vue par les experts », "Ubérisation : faut-il avoir peur ? »... Mais aussi « Vers une "ubérisation" du droit? », « Vers une ubérisation du marketing » ou même - car il n'est pas toujours employé péjorativement - "Vivement "l'ubérisation" de la vie politique française ! ». Le mot, désormais employé à toutes les sauces, apparaît déjà comme dévoyé30.

Dans la version 2017 du Petit Robert, on trouvera la définition suivante : ubériser « déstabiliser et transformer avec un modèle économique innovant tirant parti des nouvelles technologies $»^{31}$. Le Petit Larousse 2017 lui préfère ubérisation qu'il définit comme " remise en cause du modèle économique d'une entreprise ou d'un secteur d'activité par l'arrivée d'un nouvel acteur proposant les mêmes services à des prix moindres $\gg^{32}$.

\section{EN GUISE DE CONCLUSION : LE MOT CLÉ DU CHANGEMENT CIVILISATIONNEL}

Si nous avions à choisir le mot qui signifie le mieux le changement de ces dernières années, ce serait ère numérique. On est déjà habitué à lire des tweets des hommes politiques devenus un support de plus pour faire circuler leurs réactions

${ }^{30} \mathrm{https} / / /$ www.uberisation.org/fr/portfolio/luberisation-de-la-soci $\% \mathrm{C} 3 \% \mathrm{~A} 9 \mathrm{t} \% \mathrm{C} 3 \% \mathrm{~A} 9-\mathrm{d} \% \mathrm{C} 3 \%$ A9finition (dernière consultation : le 30.12.2017).

${ }^{31}$ Ibid.

${ }^{32}$ Ibid. 
et leurs commentaires sur l'actualité politique. Rappelons qu'Édouard Philippe, le premier ministre français, a tweeté son commentaire sur le conseil des ministres en se déplaçant dans Paris sur un vélib ${ }^{33}$.

De nombreux ouvrages scientifiques traitent le phénomène du numérique sous un angle différent. On parle de la révolution numérique, de la société numérique, de la transformation, de la conversion qui s'est opérée grâce à la technologie numérique, etc. L'impact de l'informatique est visible, on peut le dire sans exagérer, dans tous les domaines y compris le domaine culturel, scientifique et même, elle s'est introduite dans l'analyse du discours. Citons le dernier ouvrage de M.-A. Paveau L'analyse du discours numérique (2017). Elle $\mathrm{y}$ introduit les notions de technodiscours, technomot, technosigne, technogenre de discours, technographisme, etc. Cette nouvelle réalité affirme que "les discours numériques natifs ne sont pas d'ordre purement langagier » qu'ils sont déterminés par les techniques du numérique, ce qui montre le besoin de modifier l'analyse traditionnelle du discours (ibid., p. 11).

Nous voulons conclure avec cette remarque que nous avons profité abondamment des bons côtés du numérique en écrivant cet article.

\section{Références bibliographiques}

\section{Ouvrages}

HADDAD, Raphä̈l (dir.) (2017), Manuel d'écriture inclusive. Faites progresser l'égalité femmes/ hommes par votre manière d'écrire, Paris, Agence de communication d'influence Mots-Clés, www.ecriture-inclusive.fr.

KEYES, Ralph (2004), The Post-Truth Era: Dishonesty and Deception in Contemporary Life, New York, St. Martin's Press.

PAVEAU, Marie-Anne (2017), L'analyse du discours numérique. Dictionnaire des formes et des pratiques, Paris, Hermann.

\section{Dictionnaires}

CNRTL : www.cnrtl.fr.

$T L F i$ : www.atilf.atilf.fr.

WIKTIONARY : www.fr.wiktionary.org.

\footnotetext{
${ }^{33} \mathrm{http}$ ://www.canalplus.fr/emissions/pid8896-1-info-du-vrai.html?vid=1464896 (dernière consultation : le 30.12.2017).
} 


\section{Sitographie}

www.bfmtv.com/politique/texte-le-discours-d-emmanuel-macron-au-congres-de-versailles1200523.html.

www.canalplus.fr/emissions/pid8896-1-info-du-vrai.html?vid=1464896.

www.fr.wikipedia.org/wiki/\%C3\%88re post-v\%C3\%A9rit\%C3\%A9.

www.fr.wikipedia.org/wiki/Hoax.

www.franceinter.fr/emissions/l-invite-de-8h20/1-invite-de-8h20-22-decembre-2017.

www.lefigaro.fr/actualite-france/2017/03/06/01016-20170306ARTFIG00187-fake-news-unmeme-terme-pour-plusieurs-realites.php.

www.lefigaro.fr/langue-francaise/actu-des-mots/2017/11/02/37002-20171102ARTFIG00264-fakenews-elu-mot-de-1-annee-par-le-dictionnaire-collins.php.

www.lemonde.fr/les-decodeurs/article/2017/01/25/faits-alternatifs-fake-news-post-verite-petitlexique-de-la-crise-de-l-information_5068848_4355770.htm.

www.lesechos.fr/16/11/2016/lesechos.fr/0211500050190_selon-les-dictionnaires-oxford--le-motde-1-annee-est---.htm.

www.motdelannee.fr.

www.next.liberation.fr/culture-next/2016/11/16/post-verite-mot-de-1-annee-du-dictionnaireoxford_1528775.

www.rts.ch/info/monde/8551111-l'influences-des-fake-news-sur-la-presidentielle-francaise-seraitreelle-html.

www.uberisation.org/fr/portfolio/luberisation-de-la-soci\%C3\%A9t\%C3\%A9-d\%C3\%A9finition. 